\title{
Influences on human papillomavirus
} (HPV)-related information needs among women having HPV tests for follow-up of abnormal cervical cytology

\author{
Mairead O'Connor, ${ }^{1}$ Liza Costello, ${ }^{2}$ Judith Murphy, ${ }^{3}$ Walter Prendiville, ${ }^{4}$ \\ Cara M Martin, ${ }^{5}$ John J O'Leary, ${ }^{6}$ Linda Sharp, ${ }^{7}$ on behalf of the Irish \\ Screening Research Consortium (CERVIVA)
}

- Additional material is published online only. To view please visit the journal online (http://dx.doi.org/10.1136/jpprhc2013-100750).

For numbered affiliations see end of article.

\section{Correspondence to} Dr Mairead O'Connor, National Cancer Registry Ireland, Building 6800, Cork Airport Business Park, Kinsale Road, Cork, Ireland;

m.oconnor@ncri.ie

Received 7 August 2013 Revised 9 June 2014 Accepted 15 August 2014 Published Online First 23 September 2014
CrossMark

To cite: O'Connor M, Costello L, Murphy J, et al. J Fam Plann Reprod Health Care 2015;41:134-141.

\begin{abstract}
Objectives Testing for human papillomavirus (HPV) infection has recently been introduced into cervical screening programmes. We investigated (1) barriers to accessing and absorbing information and (2) factors that influence information needs among women undergoing HPV tests.
\end{abstract}

Methods In-depth interviews were conducted with 27 women who had HPV tests performed in a colposcopy clinic as part of follow-up of lowgrade abnormal cytology or post-treatment for cervical intraepithelial neoplasia (CIN). Interviews were transcribed verbatim, coded and analysed using Framework Analysis, to identify main themes and sub-themes.

Results Among these women, barriers to accessing and absorbing HPV information were: being overwhelmed with information; context of the HPV test; colposcopy clinic experience(s); women's perceptions of medical professionals' behaviours and attitudes, and information available on the Internet. Factors influencing women's HPV information needs were: concerns surrounding abnormal cytology or diagnosis of CIN; amount of information provided about HPV; awareness HPV is sexually transmitted; previous negative health care experience(s); and the HPV test in relation to other life events. The timing of delivery of HPV information was key to women absorbing or remembering the information given; it was important that information was given in stages rather than altogether.

Conclusions In women undergoing HPV testing during follow-up, the amount and timing of delivery of HPV information requires careful consideration. Significant barriers exist to accessing and absorbing HPV information which,

\section{Key message points}

Several significant barriers exist to accessing and absorbing human papillomavirus (HPV) information among women undergoing HPV testing for follow-up.

- Specific factors can influence women's HPV information needs. These factors need to be taken into account when providing women with information on HPV.

- Given the likely use of primary HPV testing in the future, further research on HPV-related information issues (e.g. timing of delivery of information) is urgently required.

unless addressed, could have serious implications in terms of women's comprehension of HPV tests. Given the expanding use of HPV testing within cervical screening, further research on HPV-related information issues is needed.

\section{INTRODUCTION}

The relationship between 'high-risk' strains of human papillomavirus (HPV) and cervical lesions has stimulated considerable interest in the role of HPV testing in cervical screening. ${ }^{1}{ }^{2}$ Three possibilities exist for implementing HPV testing: (1) as a primary screening test; (2) for triage of women with low-grade abnormal cytology; or (3) in follow-up of 
women treated for cervical intraepithelial neoplasia (CIN). ${ }^{3}$ Incorporating HPV testing into screening is likely to be both effective and cost-effective $e^{4}$ and, while it is currently being introduced into screening programmes, mainly for triage or following treatment, ${ }^{67}$ it is generally accepted that in the future, HPV, rather than cytology, will be the primary screening test. ${ }^{8} 9$

From a clinical perspective, testing for HPV is straightforward and, from the woman's perspective, is identical to having a cytology test. However, studies suggest HPV testing can be a sensitive issue for women, predominately because high-risk strains are sexually transmitted. ${ }^{10}{ }^{11}$ HPV knowledge levels among women are low ${ }^{12}$ and women frequently have unanswered questions ${ }^{13} 14$ and desire more information on areas such as viral types and implications for sexual relationships. ${ }^{15} 16$

However, the current evidence base has limitations. Some studies asked women to think hypothetically about having HPV tests. ${ }^{17}$ Others explored views of women who underwent testing, but were conducted before HPV vaccinations and vaccination programmes were introduced, events which may have impacted on information needs. ${ }^{15} 18$ To date, the primary focus has been information needs per se: research has focused on the fact that women have many unanswered questions about HPV. ${ }^{13} 14{ }^{19}$ No studies appear to have examined what influences women's information needs. Moreover, little is known about the barriers to women accessing and absorbing HPV information.

We conducted in-depth interviews among women with abnormal cytology who had HPV tests as part of routine management to investigate: (1) barriers to accessing and absorbing HPV information and (2) factors that influence HPV information needs.

\section{METHODS}

\section{Study design and participants}

The study setting was Ireland where a national cervical screening programme, offering free cervical cytology tests to women aged 25-60 years commenced in $2008 .^{20}$ In 2010, a school-based HPV vaccination programme began offering free vaccination to girls aged $12-13$ years. ${ }^{21}$ The study was conducted between January and June 2011, prior to the introduction of HPV testing in the post-treatment setting within the national screening programme in 2012.

Participants were recruited through a colposcopy clinic in a public hospital. Clinic administrative staff identified 200 women who had had a HPV DNA test in the previous 6 months following one or more lowgrade cytology tests or treatment for CIN. Women were stratified by the test result; the most recent 100 women to have tested HPV-positive and the most recent 100 women to have tested HPV-negative were sent study invitation letters signed by the lead clinician (WP). Interested women $(n=45)$ returned a reply slip to the research team, and an interviewer (MO'C or LC) contacted them in the order in which they responded to arrange an interview.

\section{Ethical approval}

Ethical approval was obtained from the joint Research Ethics Committee of St James Hospital and the Adelaide and Meath Hospital, Dublin. All study participants provided written informed consent.

\section{Interviews}

In-depth, face-to-face interviews were carried out, usually in the participant's home. Interviews were guided by a semi-structured topic guide (see online supplementary Table S1), developed from a literature review, which covered: screening history; understanding of HPV and cytology results; emotional impact of the HPV test result; and HPV information needs. The point at which HPV was initially raised varied between interviews, depending on how women discussed their experiences. For some questions there were alternative versions according to the woman's HPV test result. Interviews lasted 25-70 minutes and were audio-recorded with the participants' consent. Interviews ceased once data saturation was reached (i.e. no new issues arose from the data ${ }^{22}$ ); this was achieved once 27 interviews had been conducted.

\section{Analysis}

Interviews were transcribed verbatim and anonymised. Recruitment/interviews and analysis occurred simultaneously so that issues that arose in earlier interviews could inform subsequent interviews. To ensure validity of coding and analytic rigour, the interviewers independently reviewed and coded transcripts of the first two interviews, discussed these to reach consensus, and combined codes into initial themes. These codes were applied to the remaining interviews, with code lists refined as analysis progressed. Framework Analysis was used to organise the data and identify emerging themes. ${ }^{23}$ Data were summarised within the framework to facilitate systematic organisation of the data and examination of themes. The main themes and subthemes on information derived from the data are presented. Direct quotes that illustrate the narrative accounts are presented in Tables 2 and 3. Each quote is followed by the relevant participant ID number (PT).

\section{RESULTS}

Characteristics of the 27 participants are summarised in Table 1. Eleven women stated they had tested HPV-negative, six women that they were HPV-positive and 10 women could not recall their result. 
Table 1 Study participants' characteristics $(n=27)$

\begin{tabular}{lr}
\hline Characteristic & $\boldsymbol{n}$ \\
\hline Age (years) & \\
$<30$ & 4 \\
$30-39$ & 15 \\
$40-49$ & 6 \\
$\geq 50$ & 2 \\
Marital status & \\
Single & 5 \\
In a relationship, not cohabiting & 4 \\
Cohabiting & 3 \\
Married & 12 \\
Divorced/separated & 2 \\
Widowed & 1 \\
Education level & 1 \\
Secondary level: lower & 6 \\
Secondary level: upper & 6 \\
Tertiary: non degree & 14 \\
Tertiary: degree & \\
Self-reported HPV test result & 10 \\
HPV negative & 11 \\
HPV positive & \\
Could not recall result & \\
\hline HPV human papillomavirus. & \\
& \\
\hline
\end{tabular}

\section{Women's HPV knowledge}

HPV knowledge levels were low. Some women had never heard of HPV before their clinic visit. Among those who had heard of it, a few knew that it was a common infection, were aware of the sexual nature of transmission, and its link to cervical cancer. Women perceived that public awareness of HPV was low.

\section{Barriers to accessing and absorbing HPV information}

Five themes relating to barriers to accessing and absorbing HPV information emerged: (1) being overwhelmed with information; (2) context of the HPV test; (3) experience(s) of attending the colposcopy clinic; (4) women's perceptions of medical professionals' behaviours and attitudes; and (5) information available on the Internet (Table 2).

Overwhelmed with information

Some women felt they had received a lot of information during their follow-up: they described feeling overwhelmed with information about abnormal cytology, colposcopy and treatment. Consequently, it was difficult for them to absorb additional information on HPV. Women described needing time to digest information received, which meant that they did not ask questions about HPV. Information overload impaired some women's ability to process and understand information they had received regarding HPV. As a result, some women were uncertain if they had received information about HPV while others forgot the details.
Context of the HPV test

A small number of women thought that the test was for research and were happy to have it unquestioningly; this prevented them from enquiring any further about HPV or the test. Others perceived the test as part of normal follow-up, which also acted as a barrier to them asking questions about HPV. Most participants described undergoing colposcopy or treatment as unpleasant, and wanted the procedure(s) over as quickly as possible. This served as a barrier to asking questions and understanding the HPV information provided.

Experience(s) of attending the colposcopy clinic

Most women described their clinic experience(s) as very positive. Consequently, they had a high level of trust in the staff, which led them to have a lack of curiosity about the HPV test and not feel the need to ask questions. Others described the clinic as being "too busy"; they were conscious that other women were waiting and described not wanting to hold these women up by asking questions. While some women felt that they could telephone the clinic with questions, others felt that they could not, because it was difficult to get to speak with a medical professional.

Women's perceptions of medical professionals' behaviours and attitudes Some women felt that they would be burdening medical professionals by asking them about HPV. One interviewee described the professional as doing her "a favour" in performing the test and, because of this, did not want to ask questions. For some, their perceptions of medical professionals' attitudes and responses they received to other questions prevented them from asking questions about HPV. One woman described the nurse doing the test as having been frustrated with her for asking questions. The way in which the test was described by medical professionals prevented women from asking questions. For example, some women reported that the HPV test was described to them as "not a major deal", which meant they felt no desire to seek out further information.

For some women, the medical professional delivering information about HPV was important. Some preferred to receive information from a doctor, perceiving them as more qualified, and viewing them as a more trusted information source. Since nurses performed many colposcopies, this was a barrier to asking HPV questions. A few women preferred receiving information from nurses as they felt nurses were more "empathic" than doctors.

Information available on the Internet

Women described using the Internet to seek information about HPV. Some observed that they had done this despite having previous negative experiences of searching for other health-related information. These previous negative experiences meant that women were sceptical about websites they had previously visited. Some women were unable to understand or absorb HPV 
Table 2 Barriers to accessing and absorbing information about human papillomavirus

\begin{tabular}{lll}
\hline Barrier & Sub-theme & Sample quotes \\
\hline Overwhelmed with information & Information overload & "Sometimes when you're in there, you forget you're getting so much \\
information you forget to ... sometimes you'll often walk out and go, & 'Oh, maybe / should have asked him [the doctor] that'." [PT31]
\end{tabular}

Needing time to 'digest' information

Forgetting information received

Context of the HPV test

HPV test for research

HPV test as part of normal follow-up

Wanting examination over quickly

Positive clinic experience

colposcopy clinic
"Well I kind of felt I just needed to process it [information], and well to just let it sink in." [PT03]

"I need to write things down. If I don't write it down I just can't remember. I can remember there and then, and then I'll go home and say, 'What did they say again?'. " [PT44]

"I just knew it [HPV test] was for some kind of research, so that much, give them a bit of time, no problem you know." [PT44]

"It [HPV test] wasn't kind of pinpointed as a separate thing. " [PT3]

"You're in a different frame of mind. So you just kind of go, 'get me out of here as quick as you can'." [PT44]

"You're totally comfortable straight away, so the environment is nearly second to that for me you know, they're welcoming and you know, lovely, great, all the more to make you feel comfortable and at home I, sort of didn't think beyond that like, oh gosh you know I might have cancer cells or what is HPV? I didn't even think of that." [P035]

Clinic too busy/other women waiting

Cannot ring clinic afterward

Not wanting to burden medical professional with questions

Women's perceptions of medica attitudes

Attitudes/responses of medical professionals to questions

The way in which the HPV test is presented by the medical professional

Preference for who delivers the information

Information available on the Internet Previous negative experiences of searching for information

Contradictory/exaggerated information

"Scare-mongering" health forums
"You feel as if you're holding up everybody else so you don't want to kind of [ask questions] because I mean it is busy but when you're in there." [PT20]

"Because you can't get through to them on the phone when you ring anytime, no / wouldn't start ringing them to [to ask questions]. " [PT19] "You don't want to you know, be holding up their [doctor] time either." [PT32]

"I had lots of questions and she [the nurse] answered them but five minutes later I had forgotten so I had to ask again and she was getting, I think, a little bit, just frustrated." [PT44]

"It [HPV test] was just the way they [doctors] spoke about it you know, there wasn't, there wasn't any need to worry the way they were speaking about it." [PT20]

"I never feel like they're [nurses] confident enough to tell you everything probably because the doctor would maybe have more degrees or know more details. " [PT31]

"You'd want to be really stupid to believe everything you read on the Internet, even about early pregnancy." [PT40]

"You just you can take it too seriously as well. In that, there's so many contradicting things." [PT29]

"When you're on the internet looking up something like that, it's like if you have a pain in your head and you look up a medical book, 'Jesus I have a brain tumour'. " [PT05]

HPV, human papillomavirus.

information available on the Internet because they described the information they had accessed about HPV as contradictory or exaggerated. In particular, they felt that health forums on HPV were "scare-mongering".

\section{Factors influencing women's HPV information needs}

Five factors that influenced women's HPV information needs were identified: (1) concerns surrounding abnormal cytology or diagnosis of CIN; (2) amount of information provided about HPV; (3) awareness of HPV being sexually transmitted; (4) previous negative healthcare experience(s); and (5) HPV test in relation to other life events (Table 3 and Figure 1). Women's HPV test results did not impact on their HPV information needs.

Concerns surrounding abnormal cytology or diagnosis of CIN

Some women perceived a diagnosis of CIN and issues surrounding their abnormal cytology as very serious; 
Table 3 Factors influencing women's human papillomavirus information needs

Factor Sample quotes

Concerns surrounding abnormal cytology/ "It just wouldn't have registered. It was all about what are the cells doing. " [PT11]

diagnosis of CIN

Amount of information provided about HPV

"Well I know it can cause cancer, I know its sexually transmitted, there's so many different types of it, so I'm a bit like, ok, I still don't actually understand what it is. Is it something that you can have for years, does it affect you in other ways? Do men have it? I never kind of got any of them kind of questions answered." [PT04]

Awareness of HPV being sexually transmitted

"It kind of makes me think I probably need to know a little bit more about it ... because I have one partner

Previous negative health care experience and we live together and we're together over like maybe two and a half years ... so I don't know. " [PT13]

"Before, I was diagnosed with PCOS so I've been to see several doctors and one of the most irritating things is, especially when you're paying them, is that they're not explaining anything. Like, I had a lot of questions about the HPV thing." [PT13]

HPV test in relation to other life events

"I'd be more worried about well has this [HPV infection] been causing my miscarriages and stuff like that and I had early pregnancies you know premature babies." [PT20]

CIN, cervical intraepithelial neoplasia; HPV, human papillomavirus; PCOS, polycystic ovary syndrome.

they were preoccupied by these issues and HPV was a low priority for them. Consequently, these women had low HPV information needs. Some were fearful that they had, or could develop, cervical cancer making "get[ting] rid" of their abnormal cells a higher priority than HPV.

Amount of information provided about HPV

Regardless of the amount of information women received, many had unanswered questions about HPV. Women who described receiving more information often had more unanswered questions than those who recalled having received less information. Moreover, those who had more knowledge of HPV often had more unanswered questions.

Awareness of HPV being sexually transmitted

The association between HPV and sexual activity was not discussed unless raised by participants. Some women knew HPV was sexually transmitted from being told by a medical professional. These women had a stronger desire for HPV information than women who did not discuss, or did not appear to be aware of, the link with sexual activity.

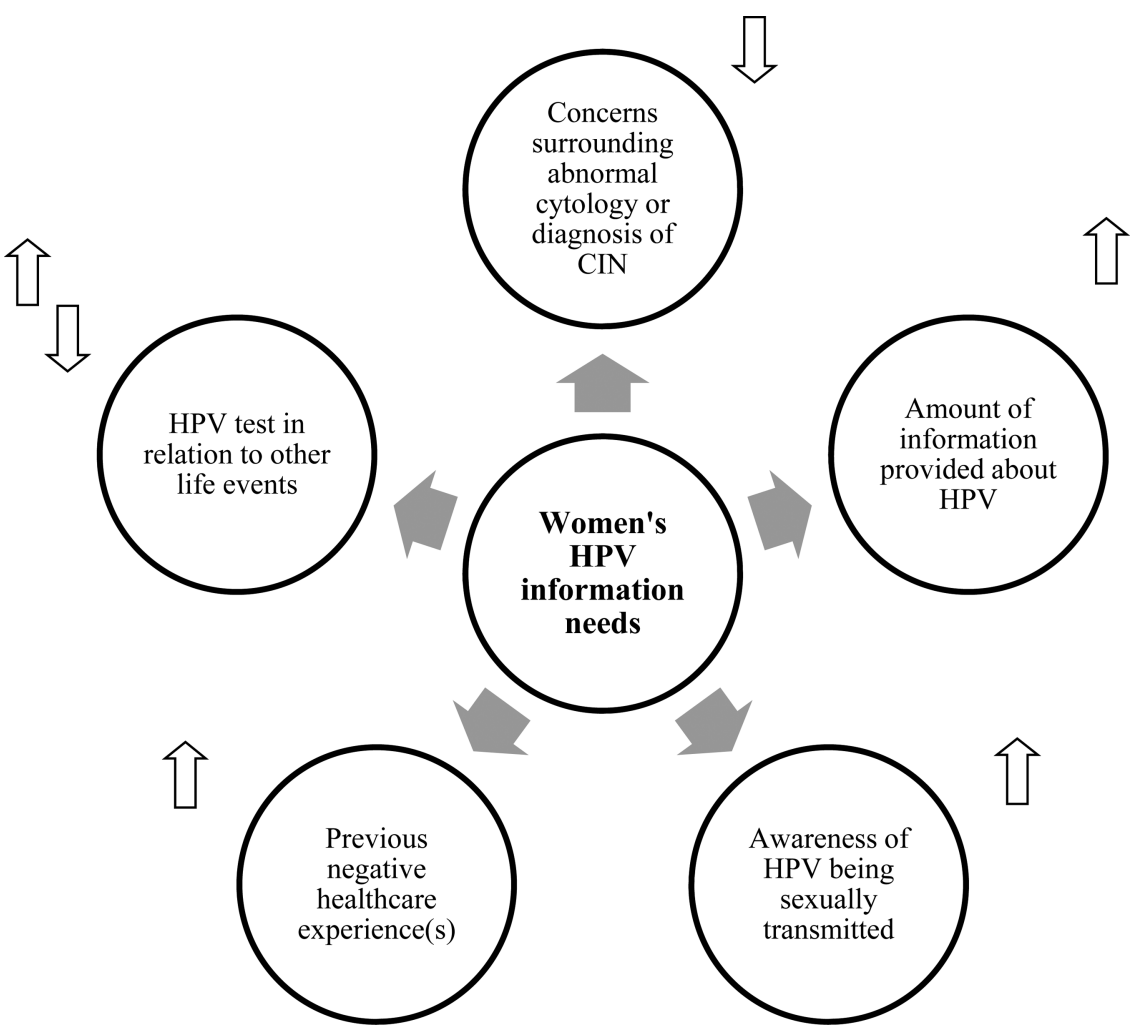

Figure 1 Factors that influence women's human papillomavirus (HPV) information needs. Arrows indicate the direction in which the factor influenced HPV information needs. Upwards arrows indicate that the factor increased needs; downward arrows that it decreased needs. CIN, cervical intraepithelial neoplasia; HPV, human papillomavirus. 
Previous negative healthcare experience(s)

Some women disclosed previous negative experiences with care received in relation to cervical screening or other gynaecological health issues. These women, in general, had high HPV information needs; they typically had more questions and/or desired more information on HPV than other women.

\section{HPV test in relation to other life events}

Some women were preoccupied by concerns other than their abnormal cytology; these women had lower HPV information needs. In contrast, for others, having a HPV test increased their information needs as this related to other issues in their lives around the time of the test. For example, when one woman learned that HPV was sexually transmitted and that she had tested HPV-positive, she felt it confirmed that her husband was being unfaithful. Another woman wondered if her HPV infection was related to her recent miscarriages.

\section{Women's suggestions for improving HPV information}

To raise awareness, women felt that, as well as information about cytology tests, HPV information should be given to women participating in screening. The timing of delivery of information to women was of paramount importance. Women suggested information about abnormal cytology and HPV be provided in stages, so as not to be overwhelming. With regard to preferred formats, some women favoured pamphlets, while others preferred face-to-face discussions with medical professionals.

\section{DISCUSSION}

This study highlights the challenges facing medical professionals and screening programmes in relation to information delivery to women undergoing HPV tests. The results suggest that in the specific context of women undergoing HPV testing in a colposcopy clinic, several barriers exist to women accessing and absorbing information about HPV and that certain factors can influence women's HPV information needs.

\section{Barriers to accessing and absorbing information}

Feeling overwhelmed by information emerged as a major barrier to women accessing, processing and understanding HPV information. Women felt inundated with information about cytology tests, colposcopy and treatment and found it difficult to remember, or process, information about HPV. Previous studies have investigated women's satisfaction with information about abnormal cytology ${ }^{24}$ and colposcopy, ${ }^{25}$ but none considered HPV information. As far as we are aware, our study is the first to identify that feeling overwhelmed with information may prevent women from absorbing information about HPV or asking questions. Similarly, research on general cancer information overload suggests that individuals stop processing and thus absorbing information before reaching an overloaded state. ${ }^{26}$ As HPV testing becomes routinely used, screening programmes need to take care not to exacerbate this situation. Given the complexities surrounding HPV infection, it is likely that it is not just the volume of HPV information that impedes women's understanding of the information they receive. Exactly how much information about HPV is useful to women and how to convey this information in a way that is understandable requires careful consideration.

Our study highlights that women's perceptions of medical professionals' attitudes can be barriers to meeting women's HPV information needs. A previous study reported women's dissatisfaction with doctors giving inadequate time to explain HPV test results ${ }^{15}$ and aspects of our findings were consistent with this. We have also shown that some women perceive themselves as inconveniencing medical professionals by seeking HPV information. Previous research also found that women may be dissatisfied with the information-giving style of medical professionals with regard to HPV. ${ }^{15}$ We have revealed that medical professionals' behaviours and attitudes when discussing HPV impacts on women's willingness to ask for more information. These findings highlight that medical professionals need to ensure women feel they can ask HPV-related questions.

Since 2008, the number of nurses performing colposcopies has increased, ${ }^{7}$ making it likely that more women will see nurses rather than doctors when undergoing colposcopy. Our study indicates that the increasing role of nurses could impact negatively on women seeking HPV information, since doctors were often viewed as a more trusted information source. However, some women in our study preferred to receive HPV information from nurses. The possibility of doctors doing sessional work as well as the lack of continuity of care in hospital settings may mean that nurses are sometimes more experienced at understanding women's needs and providing women with appropriate HPV information.

\section{Factors influencing womens' HPV information needs}

It is well documented that abnormal cytology results and follow-up cause distress. ${ }^{27-29}$ Consistent with this, most women in our study were anxious about their abnormal cytology/treatment. Furthermore, women's concerns surrounding their abnormal cytology/treatment outweighed any HPV concerns and deprioritised any need for HPV information. However, it should be noted that the women had low levels of awareness of HPV, which (as we have shown) may have served to reduce their HPV information needs. These findings have implications for development of information materials and suggest that women's concerns surrounding their abnormal cytology should be addressed before providing HPV information. While screening programme providers may be reassured that, for most women, anxieties 
over cytology results take precedence over HPV, all women undergoing HPV tests still need to be adequately informed.

Timing of delivery of HPV information was crucial with regard to women's assimilation of it. Women were anxious to have procedures completed quickly but most received information about HPV, in many cases for the first time, during their appointment. Neither the context (i.e. of a gynaecological examination) nor women's anxieties surrounding procedures were conducive to absorbing HPV information. Consideration is therefore needed in assessing the most appropriate times at which to provide women with HPV information.

Studies have described a dearth of HPV information available to women. ${ }^{15} 16$ Crucially, we found that more information on HPV is not necessarily 'better'. For some women, receiving more HPV information increased their information needs further. Consistent with this, others have shown that giving women a lot of HPV information may increase uncertainty surrounding HPV. ${ }^{30}$ Screening providers need to accept that, in terms of HPV information provision, 'one size' will not fit all women. Ideally, medical professionals should assess each woman's needs and tailor HPV information to suit the woman, rather than providing the same information to all women. While the feasibility of this may be challenging, health messages, tailored to respondents' characteristics are effective in inducing behavioural change. ${ }^{31}$ In addition, research examining how medical professionals in busy colposcopy clinics can quickly but adequately assess individual HPV information needs is urgently required. In the meantime, other approaches to combat information overload might be considered, taking into account women's suggestions about providing information in stages. Examples include providing women with references to reputable websites after their colposcopy, or giving them written information which they could take away to read at a later stage.

\section{Study strengths and limitations}

To our knowledge, our study is the first to focus specifically on barriers to accessing and absorbing HPV information and factors influencing information needs of women undergoing HPV tests. A major strength is that the study was conducted among women who actually had HPV tests as triage or post-treatment follow-up. However, we were unable to determine whether barriers and factors influencing information needs varied between women tested for triage or as part of posttreatment follow-up. Moreover, given that primary HPV testing is likely to be used in the future, the information needs of women undergoing HPV testing in that specific context warrants additional research.

A limitation is that the study was conducted in one colposcopy clinic. However, the clinic is one of the 15 colposcopy clincs in Ireland affiliated with the screening programme; these clinics provide standardised, quality-assured care. A maximum variation sample was recruited: participants' socio-demographic characteristics were diverse and both HPV-positive and HPV-negative women were interviewed. While the sample size $(n=27)$ is not large in absolute terms, data saturation in the group had been reached by this point. As such, the sample size was sufficient for the study purpose. ${ }^{32}$ Another limitation is that most women had tertiary education and are thus over-represented. However, since health literacy is higher among those with higher education, ${ }^{33}$ our finding that there are significant barriers to meeting women's HPV information needs in this study population is striking.

\section{CONCLUSIONS}

For women undergoing HPV tests for follow-up of abnormal cytology or treatment, the amount and timing of delivery of HPV information needs careful consideration. The barriers to accessing and absorbing HPV information in this context - particularly in relation to information overload - need to be addressed in order to increase women's comprehension of HPV tests. The factors identified as impacting on women's HPV information needs in this context, in particular concerns surrounding abnormal cytology, should be considered when providing women with information on HPV. More research on HPV-related information issues (e.g. timing of delivery of information) is required, especially given the expanding use of HPV testing in triage and post-treatment follow-up and its likely introduction as a primary screening test in the future.

\section{Author affiliations}

${ }^{1}$ Study Coordinator, National Cancer Registry Ireland, Cork, Ireland

${ }^{2}$ Study Researcher, National Cancer Registry Ireland, Cork, Ireland

${ }^{3}$ Study Coordinator, National Cancer Registry Ireland, Cork, Ireland

${ }^{4}$ Associate Professor and Consultant in Obstetrics and Gynaecology, The Adelaide and Meath Hospital, Dublin, Ireland

${ }^{5}$ Assistant Professor in Molecular Pathology and Tumour Biology, Coombe Women and Infants University Hospital, Dublin, Ireland

${ }^{6}$ Professor of Pathology, Coombe Women and Infants University Hospital, Dublin, Ireland ${ }^{7}$ Epidemiology and Adjunct Professor, National Cancer Registry Ireland, Cork, Ireland

Acknowledgements The authors thank the colposcopy clinic staff at the Adelaide and Meath Hospital, Dublin and the women who participated in the study. They are also grateful to the administrative staff for transcribing the interviews.

Funding This study was undertaken as part of the CERVIVA research consortium (http://www.cerviva.ie). CERVIVA is funded by the Health Research Board, Ireland. 


\section{Competing interests None.}

Ethics approval Joint Research Ethics Committee of St James Hospital and the Adelaide and Meath Hospital, Dublin.

Provenance and peer review Not commissioned; externally peer reviewed.

\section{REFERENCES}

1 Bosch FX, Lorincz A, Muñoz N, et al. The causal relation between human papillomavirus and cervical cancer. J Clin Pathol 2002;55:244-265.

2 International Agency for Research on Cancer (IARC). IARC Handbooks of Cancer Prevention. Volume 10: Cervix Cancer Screening. Lyon, France: IARC Press, 2005.

3 Cuzick J, Arbyn M, Sankaranarayanan R, et al. Overview of human papillomavirus-based and other novel options for cervical cancer screening in developed and developing countries. Vaccine 2008;26:29-41.

4 Arbyn M, Ronco G, Anttila A, et al. Evidence regarding human papillomavirus testing in secondary prevention of cervical cancer. Vaccine 2012;30:F88-F99.

5 Vijayaraghavan A, Efrusy MB, Mayrand MH, et al. Cost-effectiveness of high-risk human papillomavirus testing for cervical cancer screening in Québec, Canada. Can J Public Health 2010;101:220-225.

6 Department of Health. Improving Outcomes: A Strategy for Cancer. 2011. http://www.dh.gov.uk/prod_consum_dh/groups/ dh_digitalassets/documents/digitalasset/dh_123394.pdf [accessed 18 January 2013].

7 National Cervical Screening Programme (CervicalCheck). CervicalCheck Programme Report 2010-2011. Ireland: National Cancer Screening Service, 2012. http://www. cervicalcheck.ie/_fileupload/CervicalCheck $\% 20$ programme $\%$ 20report $\% 201 \% 20$ Sept $\% 202010 \% 20-\% 2031 \% 20$ August $\%$ 202011\%20-\%20Final.pdf [accessed 18 January 2013].

8 Saslow D, Solomon D, Lawson HW, et al. American Cancer Society, American Society for Colposcopy and Cervical Pathology, and American Society for Clinical Pathology screening guidelines for the prevention and early detection of cervical cancer. CA Cancer J Clin 2012;62:147-172.

9 Arbyn M, de Sanjosé S, Saraiya M, et al. EUROGIN 2011 roadmap on prevention and treatment of HPV-related disease. Int J Cancer 2012;131:1969-1982.

10 McCaffery K, Waller J, Forrest S, et al. Testing for human papillomavirus in women with abnormal pap smear results. JAMA 2002;288:1350.

11 McCaffery K, Waller J, Forrest S, et al. Testing positive for human papillomavirus in routine cervical screening: examination of psychosocial impact. BJOG 2004;111:1437-1443.

12 Waller J, McCaffery K, Nazroo J, et al. Making sense of information about HPV in cervical screening: a qualitative study. Br J Cancer 2005;92:265-270.

13 Goldsmith MR, Bankhead CR, Kehoe ST, et al. Information and cervical screening: a qualitative study of women's awareness, understanding and information needs about HPV. J Med Screen 2007;14:29-33.

14 Dyson S, Pitts M, Lyons A, et al. Providing high quality information about human papillomavirus for women after treatment for high-grade cervical dysplasia. Sex Health 2010;7:49-54.

15 McCaffery K, Irwig L. Australian women's needs and preferences for information about human papillomavirus in cervical screening. J Med Screen 2005;12:134-141.
16 Anhang R, Wright TC Jr, Smock L, et al. Women's desired information about human papillomavirus. Cancer 2004;100:315-320.

17 McCaffery K, Forrest S, Waller J, et al. Attitudes towards HPV testing: a qualitative study of beliefs among Indian, Pakistani, African-Caribbean and white British women in the UK. Br J Cancer 2003;88:42-46.

18 McCaffery K, Waller J, Nazroo J, et al. Social and psychological impact of HPV testing in cervical screening: a qualitative study. Sex Transm Infect 2006;82:169-174.

19 Marlow LA, Wardle J, Grant N, et al. Human papillomavirus (HPV) information needs: a theoretical framework. J Fam Plann Reprod Health Care 2009;35:29-33.

20 National Cervical Screening Programme (CervicalCheck). CervicalCheck Programme Report 2008-2009. Ireland: National Cancer Screening Service, 2010. http://www. cervicalcheck.ie/_fileupload/File/CervicalCheck\%20Programme \%20Report\%202008-2009.pdf [accessed 10 February 2013].

21 National Immunisation Advisory Committee of the Royal College of Physicians of Ireland. Immunisation Guidelines for Ireland, 2008 Edition. Chapter 6a - Human Papillomavirus. 2011. http://www.immunisation.ie/en/HealthcareProfessionals/ ImmunisationGuidelines2008/ [accessed 10 February 2013].

22 Francis JJ, Johnston M, Robertson C, et al. What is an adequate sample size? Operationalising data saturation for theory-based interview studies. Psychol Health 2010;25:1229-1245.

23 Ritchie J, Spencer L. Qualitative data analysis for applied policy research. In: Bryman A, Burgess R (eds), Analysing Qualitative Data. London, UK: Routledge, 1994:173-194.

24 Bertram CC, Magnussen L. Informational needs and the experiences of women with abnormal Papanicolaou smears. J Am Acad Nurse Pract 2008;20:455-462.

25 Swancutt DR, Greenfield SM, Luesley DM, et al. Women's experience of colposcopy: a qualitative investigation. BMC Womens Health 2011;11:11.

26 Jensen JD, Carcioppolo N, King AJ, et al. The cancer information overload (CIO) scale: establishing predictive and discriminant validity. Patient Educ Couns 2014;94:90-96.

27 Maissi E, Marteau TM, Hankins M, et al. Psychological impact of human papillomavirus testing in women with borderline or mildly dyskaryotic cervical smear test results: cross sectional questionnaire study. BMJ 2004;328:1293.

28 Flanagan SM, Wilson S, Luesley D, et al. Adverse outcomes after colposcopy. BMC Womens Health 2011;11:2.

29 Sharp L, Cotton S, Carsin AE, et al. Factors associated with psychological distress following colposcopy among women with low-grade abnormal cervical cytology: a prospective study within the Trial Of Management of Borderline and Other Low-grade Abnormal smears (TOMBOLA). Psychooncology 2013;22:368-380.

30 Rosen NO, Knäuper B, Di Dio P, et al. The impact of intolerance of uncertainty on anxiety after receiving an informational intervention about HPV: a randomised controlled study. Psychol Health 2010;25:651-668.

31 Paul CL, Redman S, Sanson-Fisher RW. A cost-effective approach to the development of printed materials: a randomized controlled trial of three strategies. Health Educ Res 2004;19:698-706.

32 Dworkin SL. Sample size policy for qualitative studies using in-depth interviews. Arch Sex Behav 2012;41:1319-1320.

33 Paasche-Orlow MK, Parker RM, Gazmararian JA, et al. The prevalence of limited health literacy. J Gen Intern Med $2005 ; 20: 175-184$. 